دراسة بالمحاكاة لنظام المؤتمرات الفيديوية عبر الثبكة اللاسلكية مع التحقيق العملي

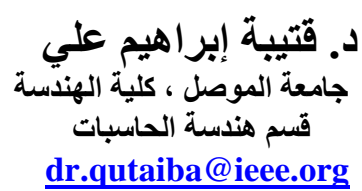

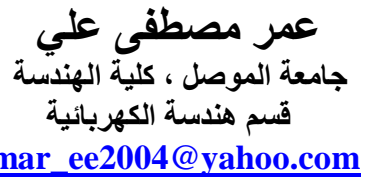

omar_ee2004@yahoo.com

\title{
الخلاصة
}

الغرض من هذا البحث هو تصميم نظام يقدم خدمات جيدة للمؤتمرات الفيديويـة عبر الثبكة المحلية اللاسلكية

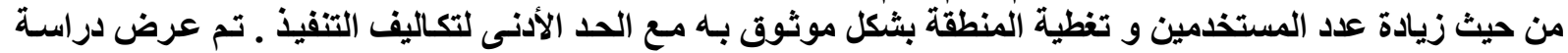

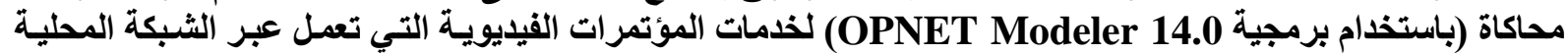
اللاسلكية (WLAN) بمعايير مختلفة (IEEE 802.11a) و (IEEE 802.11g) مع نمطين لنقل البيانـات (الأحادي

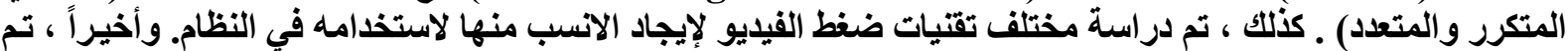

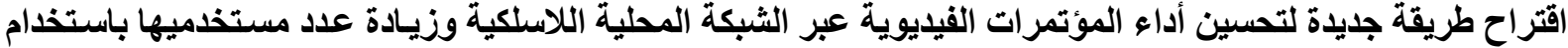

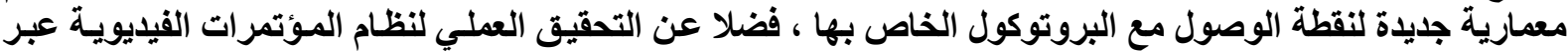

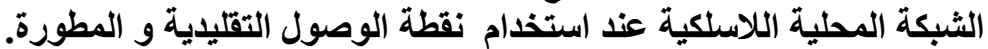
الكلمات الدالة : المؤتمرات الفيديوية ، الثبكة المحلية اللاسلكية ، النمط الأحادي المتكرر ، النمط المتعدد ، ضغط الفيديو

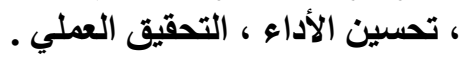

\section{A Simulation Study of Video Conference System over WLAN with Practical Implementation}

\author{
Dr. Q.A.Ali \\ Computer Engineering Department \\ College of Engineering / University of Mosul \\ O.M.Ali \\ Electrical Engineering Department
}

\section{Abstract}

The goal of the paper is to obtain a good WLAN video conference services, including an increased number of users and reliable coverage area with minimum implementation cost. We presents a simulation study ( using OPNET Modeler 14.0 package) of a video conference services working over different wireless local area network (WLAN) standards (IEEE 802.11a and IEEE 802.11g) with two different modes of data transmission (multicast and multiple unicast). Also , we examined different video compression techniques to choose a suitable one to work on the system . Finally, a new WLAN access point architecture was used to enhance video conferencing performance and to maximize the number of its users . Practical implementation of a video conferencing system was achieved over WLAN when using classic and developed Access point . 
تعد أنظمـة الشبكات المحلية اللاسلكية ( Wireless Local Area Network WLAN ) من التقنيات القات القادرة

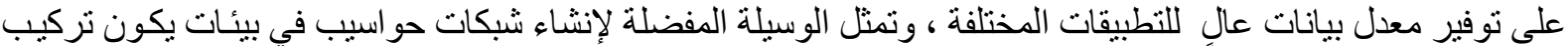

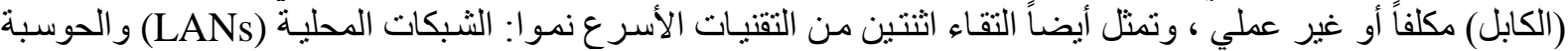

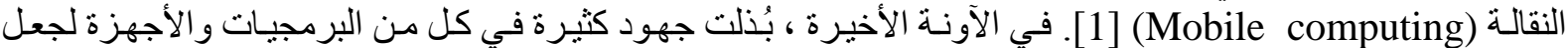

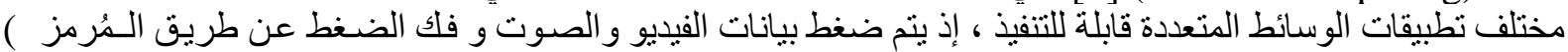

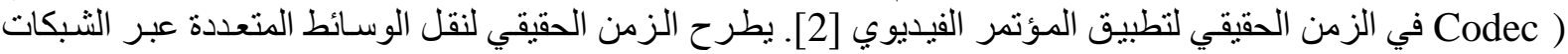

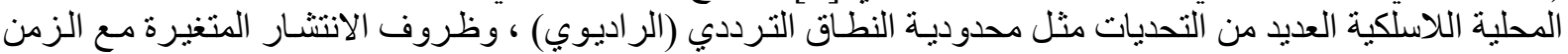

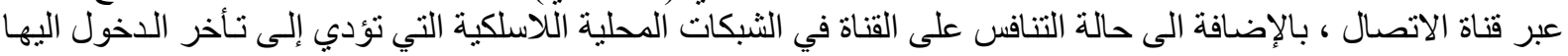

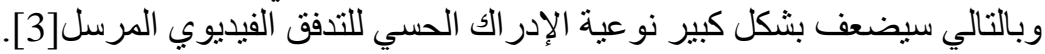

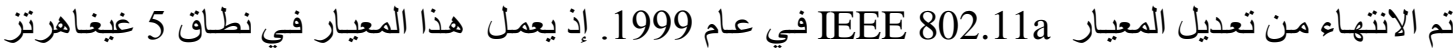
ويستخدم تقسيم التردد المتعامد لمضـاعفة الإرسـال (Orthogonal Frequency Division Multiplexing OFDM)

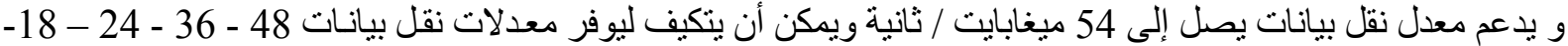

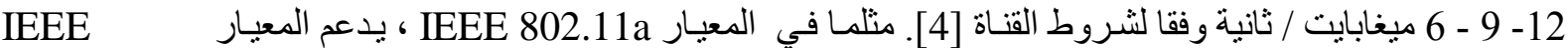

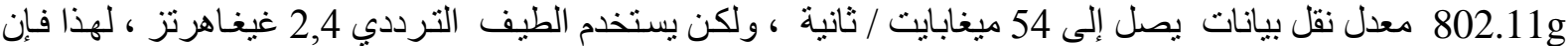

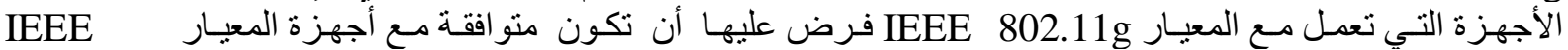

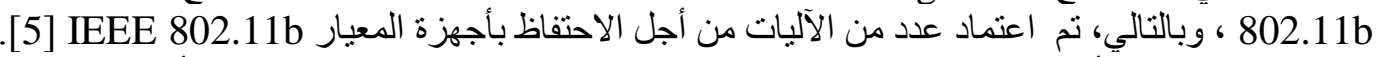

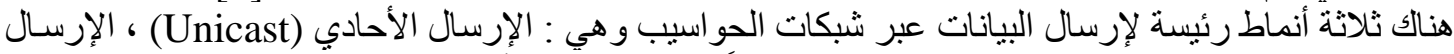

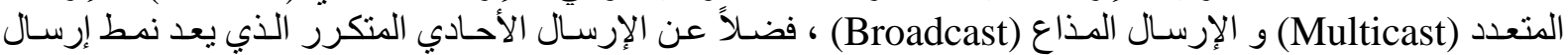

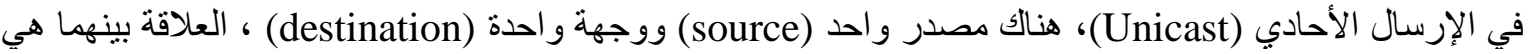

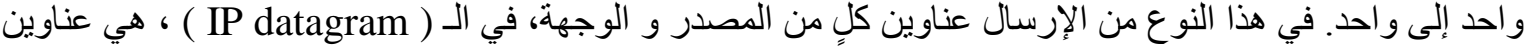
مخصصة للإرسال إلى المضيفين (hosts).

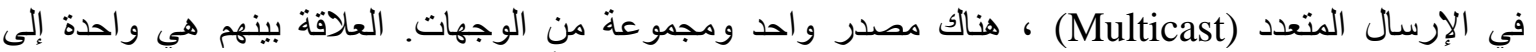

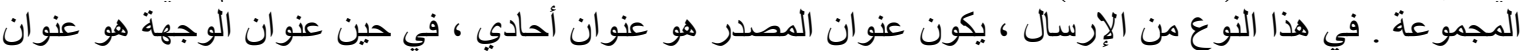

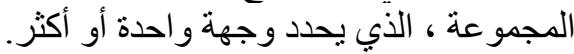

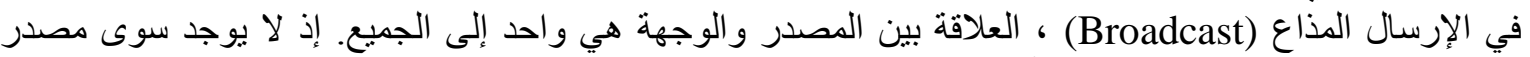
و آحد ، ولكن الوجهة هي المضيفين الآخرين جميعهم.

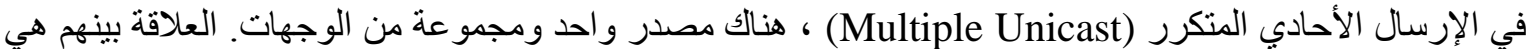

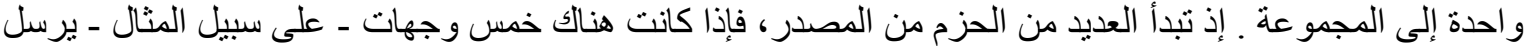

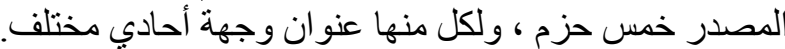

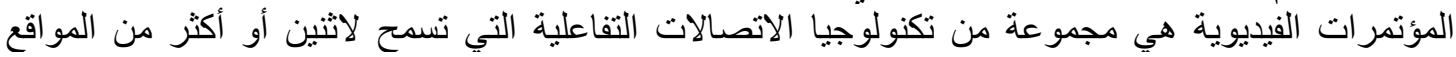

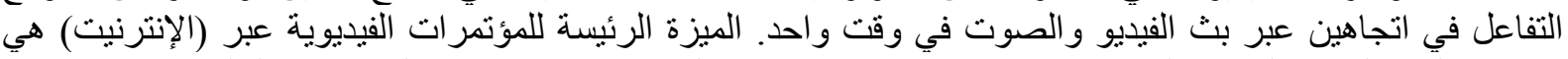

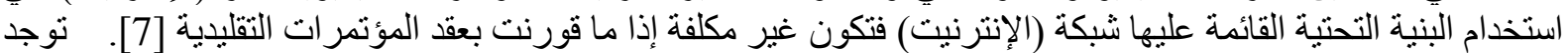

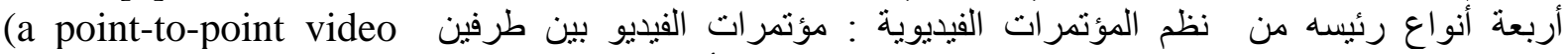

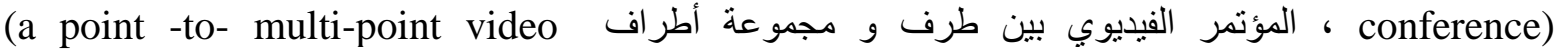

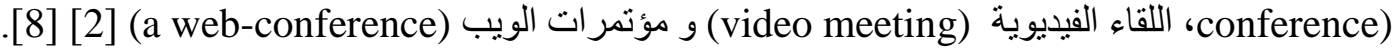
• في المؤتمر الفيديوي بين طرفين يمكن لكلا المشارِكين رؤية وسماع بعضهم البعض ، فضلا عن أنواع الفئ مختلفة من ميز ات تبادل المعلومات الخاصة. في المؤتمر الفيديوي بين طرف و و مجموعة أطر اف يمكن للطرف الرئيس ان برى ويسمع المشاركين كلهم ، و العكس

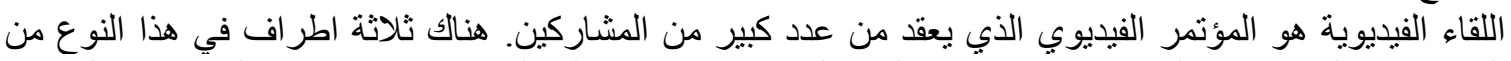

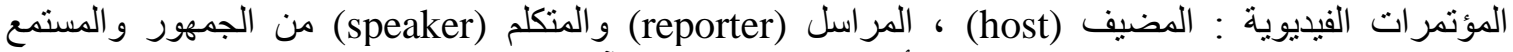
(listener)

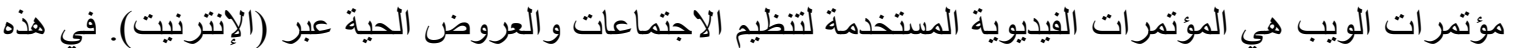

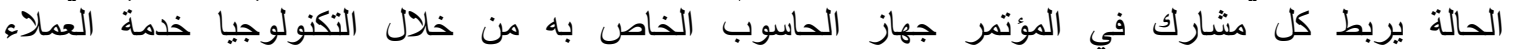
(client-server) 


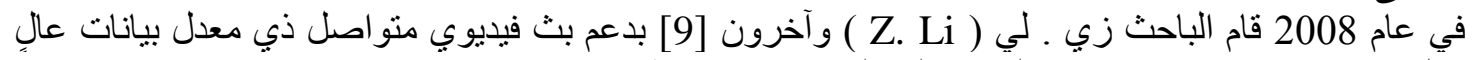

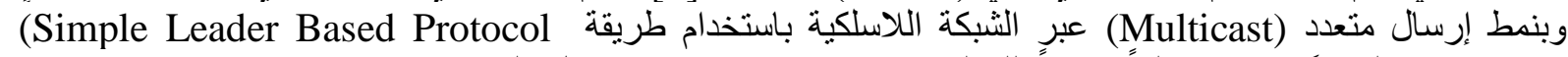

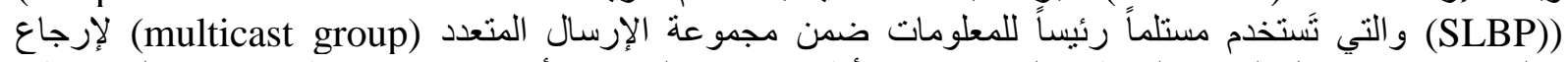

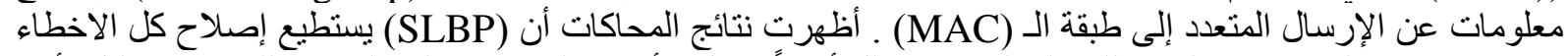

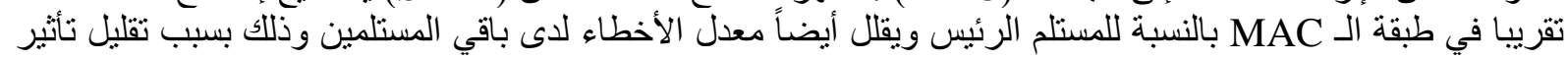

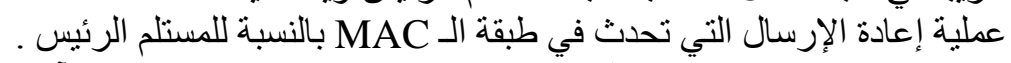

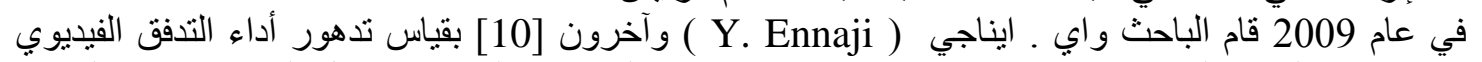

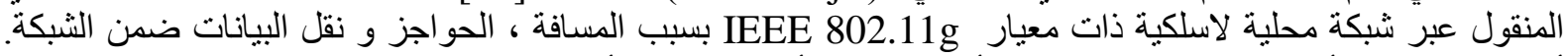

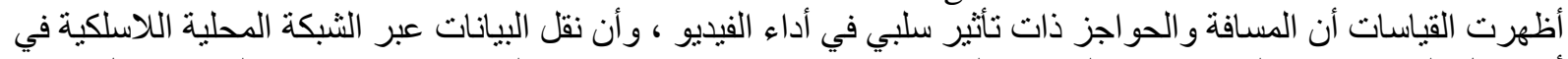

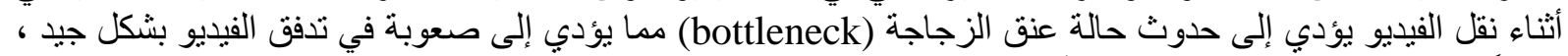

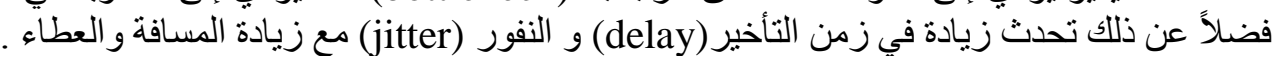

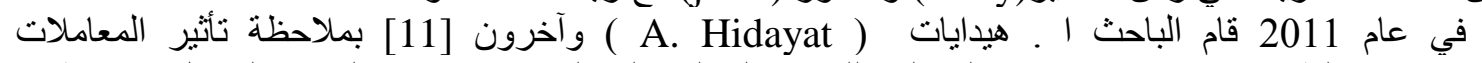

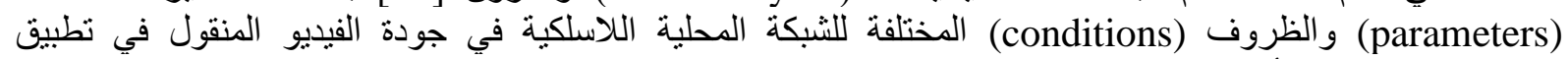

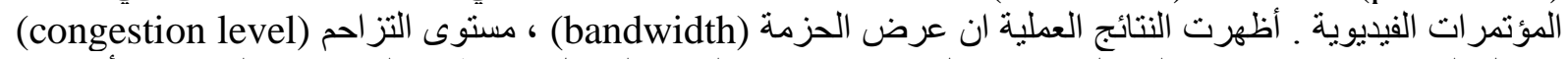

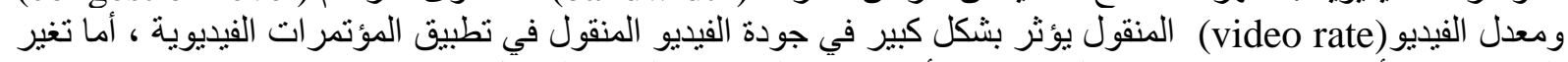
المسافة بين الأطر اف واتجاه حركتهم ليست ذات نأثير يذكر على جودة الفيديو المنقول.

\section{3- اختبار أنواع مختلفة من تقنيات ضغط الفيديو:}

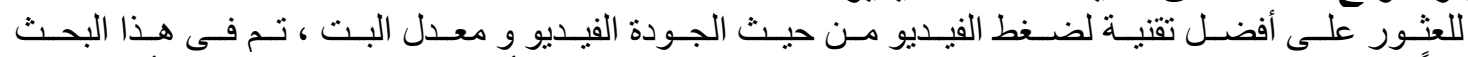

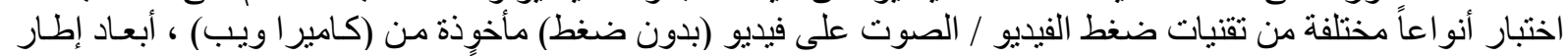

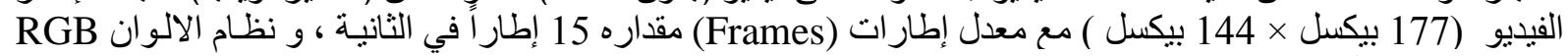

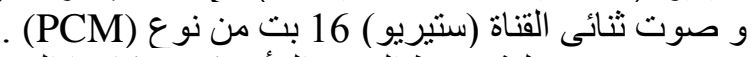

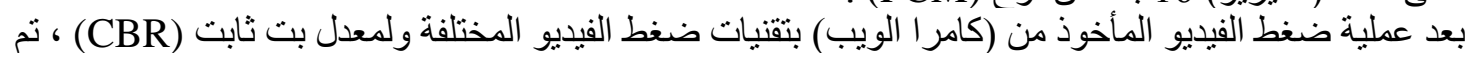

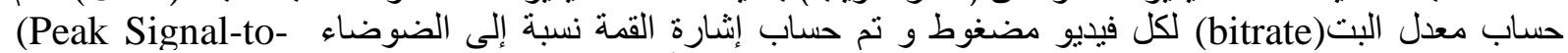
Noise Ratio PSNR)

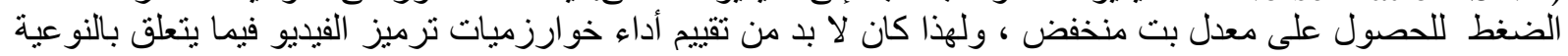
بعد البناء المتسلسل للفيديو ـ يمكن قياس نوعية الفيديو باستخدام بعض المعايير الرياضية مثل نسبة الإنشارات إلى لئي

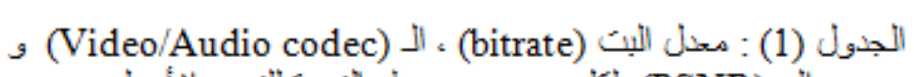

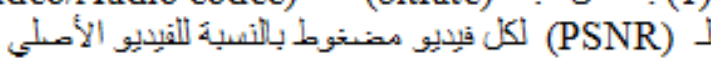

\begin{tabular}{|l|c|c|c|c|c|}
\hline \multicolumn{5}{|c|}{ Video Compression technique } \\
\hline Video Container & AV & AVI & AVI & AV & AVI \\
\hline Video Codec & RGB24 & XVD & FLV & H261 & H263 \\
\hline Video Frame Size & $176 \times 144$ & $176 \times 144$ & $176 \times 144$ & $176 \times 144$ & $176 \times 144$ \\
\hline Video Frame Rate & 15 & 15 & 15 & 15 & 15 \\
\hline Audio Format & PCM & MP3 & MP3 & MP3 & MP3 \\
\hline Fille Size / MB & 42.1 & 1.006 & 1.14 & 1.08 & 1.13 \\
\hline Overall Bit Rate / Kbps & 5793 & 135 & 157 & 149 & 156 \\
\hline Average PSNR /Y dB & 100 & 40.28 & 40.79 & 39.31 & 40.57 \\
\hline Video Container & AV & AVI & AVI & $36 P$ & RM \\
\hline Video Codec & MP4 & WMV1 & WMV3 & H263 & H.264 \\
\hline Video Frame Size & $176 \times 144$ & $176 \times 144$ & $176 \times 144$ & $176 \times 144$ & $176 \times 144$ \\
\hline Video Frame Rate & 15 & 15 & 15 & 15 & 15 \\
\hline Audio Format & MP3 & MP3 & MP3 & AMR & G.722 \\
\hline File Size / MB & 1.13 & 1.14 & 1.010 & 0.747 & 0.746 \\
\hline Overall Bit Rate / Kbps & 156 & 157 & 136 & 101 & 100.0 \\
\hline Average PSNR /Y dB & 37.86 & 41.51 & 41.19 & 32.93 & 41.81 \\
\hline
\end{tabular}

- Pink column belong to the source video

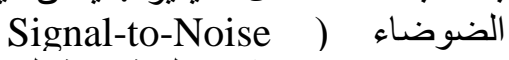
(Ratio SNR ) ، إثنارة القمة نسبة إلى (ألى

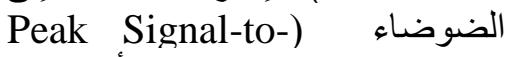
(Noise Ratio PSNR

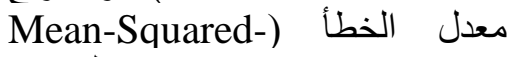
(Error MSE مجردة الإحساس فى القياس و ويرجع الفيعاير

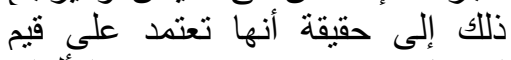

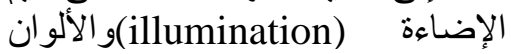

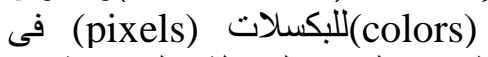

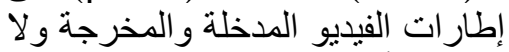

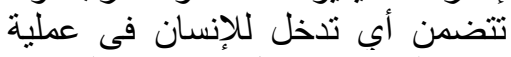
تقييم الجودة. يفضل أستخدام المقياس فئل

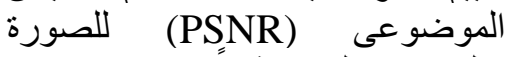

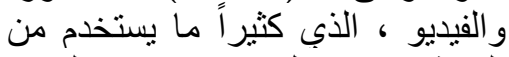
الباحثين في مجال بحوث ترميز الفيديو

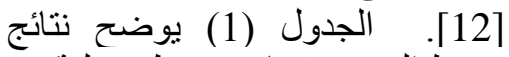
ضغط الفيديو بتقنبات ضغط مختلفة. 
4- ب بناء بيئة المحاكاة للشبكة اللاسلكية التقليدية:

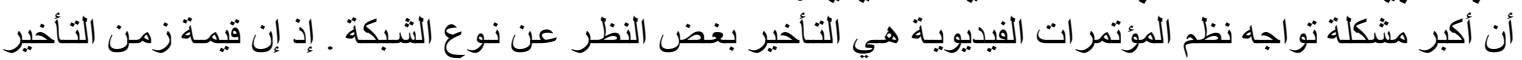

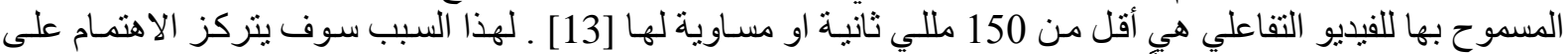

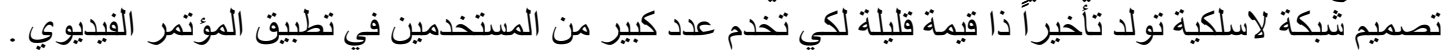

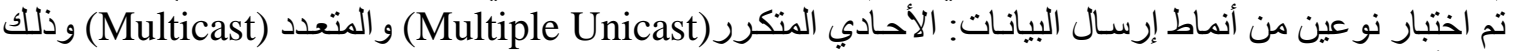

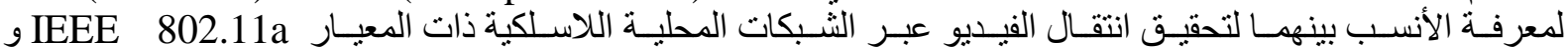

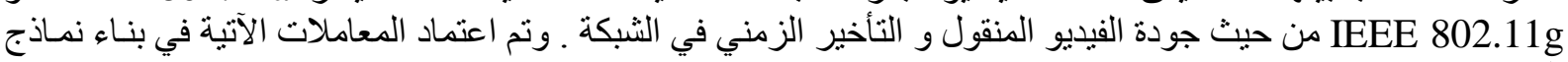

المحاكاة:

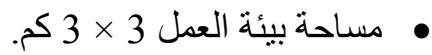

• • • استخدم نو عان من معايير الثبكة اللاسلكية في التصميم (IEEEE802.11a، (IEE2.11g).

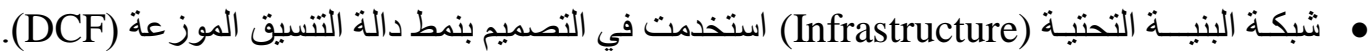

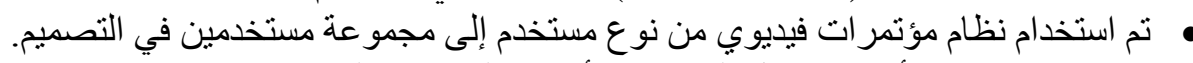

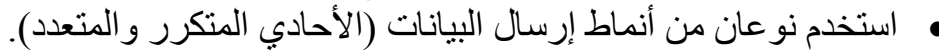

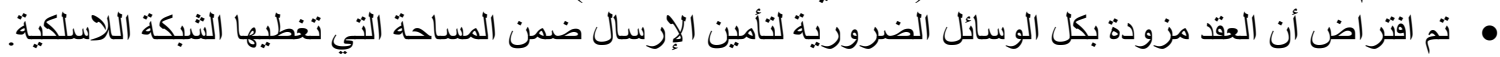

أ- الإرسال بنمط الأحادي المتكرر عبر الشبكة المحلية اللاسلكية :

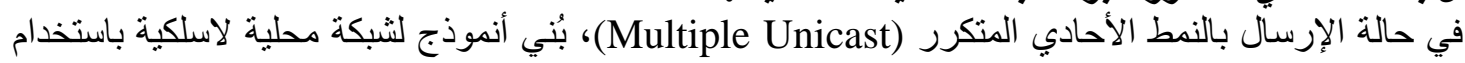

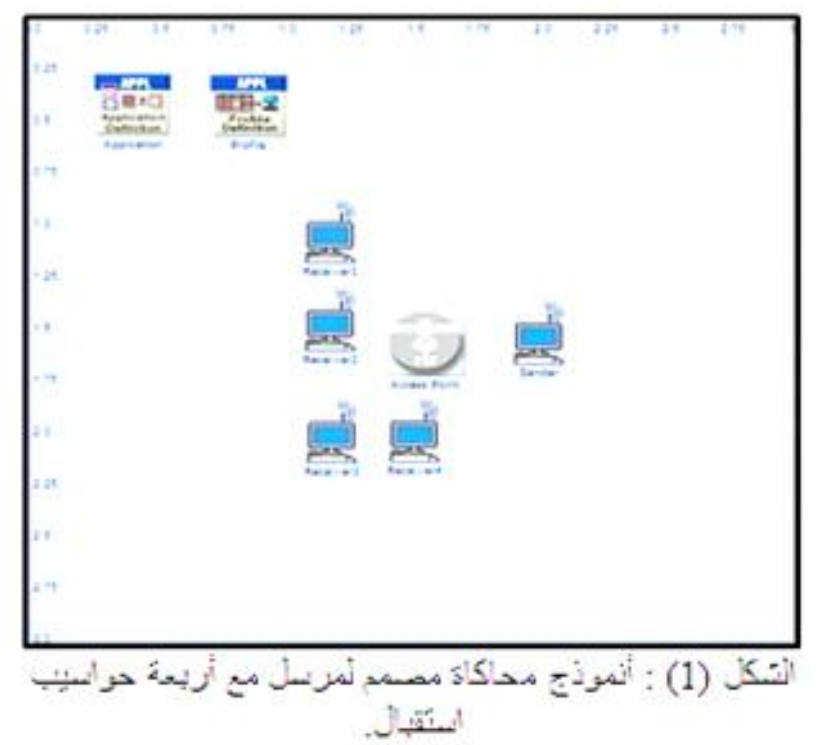

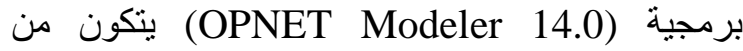

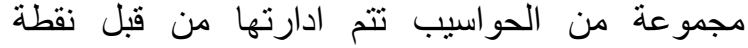

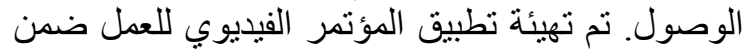

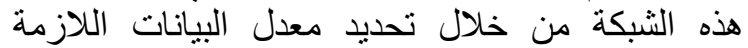

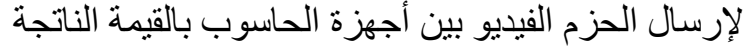

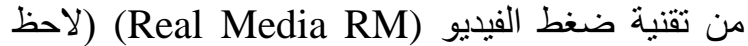
الجدول (1)). خُصص أحس الحد الحون اسيب في أنموذج الثبكة

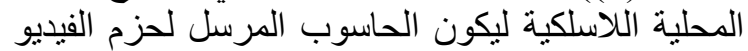

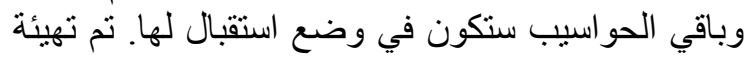

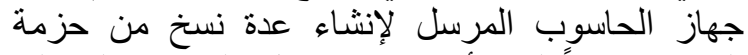

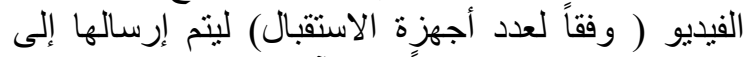

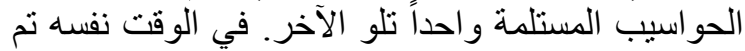

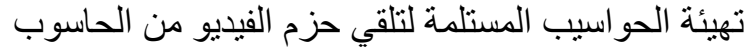

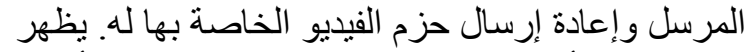

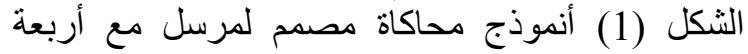
حو اسيب استقبال.

تم دراسة السعة والأداء لنظام المؤتمر الفيديوي من خلال تنفيذ المحاكاة لأنموذج مصمم لأربعة حواسيب

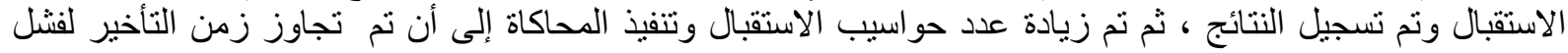

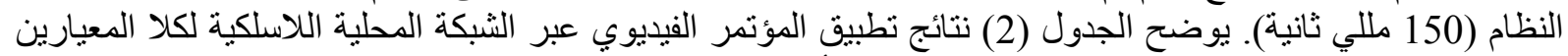
IEEE 802.11g و وبنمط الإرسال الأحادي المتكرر. IEE 8ن2.11a

بـ الإرسال بالنمط المتعدد عبر الشبكة المحلية اللاسلكية :

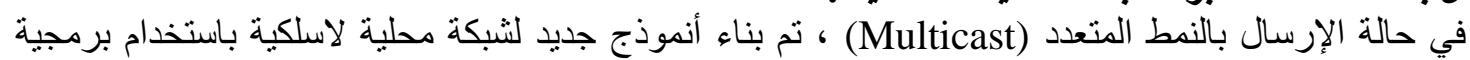

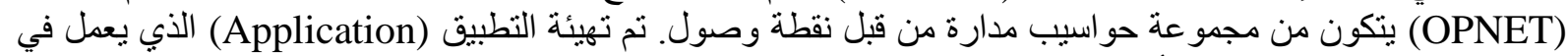

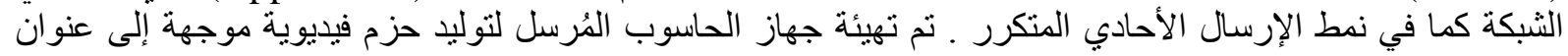

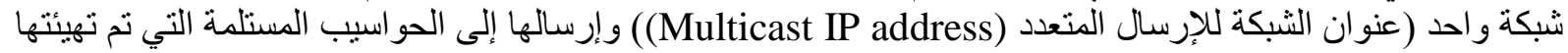
من خلال إعطاء كل من منها عنوان الثبكة نفسه للإرسال المتعدد. كما تم نهيئة نقطة الوصول لإعم الإلى الإرسال المتعدد 
علي: دراسة بالمحاكاة لنظام المؤتمرات الفيديوية عبر الثبكة اللاسلكية مع التحقيق العملي

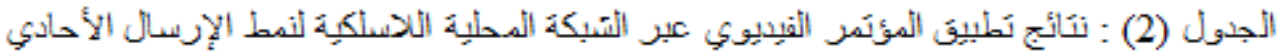
المنكرر.

\begin{tabular}{|c|c|c|c|c|c|c|}
\hline \multirow{2}{*}{ PC NO. } & \multicolumn{3}{|c|}{ IEEE 802.11a } & \multicolumn{3}{|c|}{ IEEE 802.11g } \\
\hline & $\begin{array}{c}\text { M.an Throughinut } \\
\text { (Mabps) }\end{array}$ & m.an Delay (Sec) & \begin{tabular}{|c} 
Video Conference \\
Delay (Sec)
\end{tabular} & $\begin{array}{l}\text { W.AN Throughput } \\
\text { (Altps) }\end{array}$ & W.AN Detay (Sec) & $\begin{array}{l}\text { Video Conference } \\
\text { Delay (Sec) }\end{array}$ \\
\hline $4 \mathrm{PC}$ & 0.82 & 0.00053 & 0.00053 & 0.82 & 0.00048 & 0.00048 \\
\hline $8 \mathrm{PC}$ & 1.65 & 0.00056 & 0.00056 & 1.65 & 0.00056 & 0.00056 \\
\hline $12 \mathrm{PC}$ & 2.49 & 0.00061 & 0.00061 & 2.49 & 0.00067 & 0.00067 \\
\hline $16 \mathrm{PC}$ & 3.4 & 0.00067 & 0.00067 & 3.4 & 0.0007 & 0.0007 \\
\hline $20 \mathrm{PC}$ & 4.2 & 0.0008 & 0.0008 & 4.2 & 0.00075 & 0.00075 \\
\hline $24 \mathrm{PC}$ & 5 & 0.0009 & 0.0009 & 5 & 0.0009 & 0.0009 \\
\hline $28 \mathrm{PC}$ & 5.8 & 0.00093 & 0.00093 & 5.8 & 0.00093 & 0.00093 \\
\hline $32 \mathrm{PC}$ & 6.7 & 0.0013 & 0.0013 & 6.7 & 0.001 & 0.001 \\
\hline $36 \mathrm{PC}$ & 7.5 & 0.0014 & 0.0014 & 7.5 & 0.0013 & 0.0013 \\
\hline $40 \mathrm{PC}$ & 8.2 & 0.0018 & 0.0018 & 8.2 & 0.0016 & 0.0016 \\
\hline 44 PC & 9.1 & 0.0022 & 0.0022 & 9.1 & 0.0025 & 0.0025 \\
\hline $48 \mathrm{PC}$ & 10 & 0.0027 & 0.0027 & 10 & 0.0029 & 0.0029 \\
\hline $52 \mathrm{PC}$ & 11 & 0.0032 & 0.0032 & 10.8 & 0.036 & 0.036 \\
\hline $56 \mathrm{PC}$ & 10.8 (D.B) & 0.165 & 0.165 & 10 (D. B) & 0.2 & 0.2 \\
\hline
\end{tabular}

- DB. Drop in buffer due to the over flow.

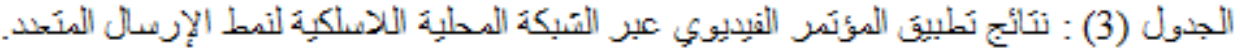

\begin{tabular}{|c|c|c|c|c|c|c|}
\hline \multirow{2}{*}{ PCNO. } & \multicolumn{3}{|c|}{ IEEE $802.11 \mathrm{a}$} & \multicolumn{3}{|c|}{ IEEE $802.11 \mathrm{~g}$} \\
\hline & $\begin{array}{c}\text { W.AN Throughput } \\
\text { (Abps) }\end{array}$ & M.AN Delay (Sec) & \begin{tabular}{|c|}
$\begin{array}{c}\text { Video Conference } \\
\text { Detay (Sec) }\end{array}$ \\
\end{tabular} & $\begin{array}{c}\text { WLAN Throughput } \\
\text { (Abps) }\end{array}$ & MLAN Delay (Sec) & \begin{tabular}{|c|}
$\begin{array}{c}\text { Video Conference } \\
\text { Delay (Sec) }\end{array}$ \\
\end{tabular} \\
\hline $4 \mathrm{PC}$ & 0.9 & 0.0018 & 0.002 & 0.9 & 0.0021 & 0.0023 \\
\hline $8 \mathrm{PC}$ & 1.7 & 0.0032 & 0.0033 & 1.7 & 0.0034 & 0.0036 \\
\hline $12 P C$ & 2.51 & 0.005 & 0.0052 & 2.51 & 0.0051 & 0.0053 \\
\hline $16 P C$ & 3.4 & 0.0063 & 0.0065 & 3.4 & 0.006 & 0.0063 \\
\hline $20 \mathrm{PC}$ & 4.3 & 0.0075 & 0.0077 & 4.3 & 0.009 & 0.0092 \\
\hline $24 \mathrm{PC}$ & 5 & 0.0084 & 0.0086 & 5 & 0.013 & 0.013 \\
\hline $28 \mathrm{PC}$ & 6 & 0.011 & 0.0115 & 6 & 0.014 & 0.014 \\
\hline $32 \mathrm{PC}$ & 6.8 & 0.013 & 0.013 & 6.8 & 0.015 & 0.015 \\
\hline $36 \mathrm{PC}$ & 7.5 & 0.015 & 0.015 & 7.5 & 0.017 & 0.017 \\
\hline $40 \mathrm{PC}$ & 8.3 & 0.019 & 0.019 & 8.3 & 0.018 & 0.018 \\
\hline $44 \mathrm{PC}$ & 9.2 & 0.02 & 0.02 & 9.2 & 0.019 & 0.019 \\
\hline $48 \mathrm{PC}$ & 10 & 0.021 & 0.021 & 10 & 0.02 & 0.02 \\
\hline $52 \mathrm{PC}$ & 11 & 0.022 & 0.022 & 11 & 0.024 & 0.024 \\
\hline $56 \mathrm{PC}$ & 11.8 & 0.025 & 0.025 & 11.8 & 0.037 & 0.037 \\
\hline $60 \mathrm{PC}$ & 12.5 & 0.026 & 0.026 & 12.5 & 0.039 & 0.039 \\
\hline $64 \mathrm{PC}$ & 13.6 & 0.027 & 0.027 & 13.6 & 0.04 & 0.04 \\
\hline $68 \mathrm{PC}$ & 14 & 0.029 & 0.029 & 13.4 (D.B) & 0.25 & 0.25 \\
\hline $72 \mathrm{PC}$ & 14.8 & 0.035 & 0.035 & & & \\
\hline $76 \mathrm{PC}$ & 16 & 0.037 & 0.037 & & & \\
\hline $80 \mathrm{PC}$ & 16.8 & 0.039 & 0.039 & & & \\
\hline $84 \mathrm{PC}$ & 16.3 (D.B) & 0.23 & 0.23 & & & \\
\hline $48 \mathrm{PC}$ & 10 & 0.0027 & 0.0027 & 10 & 0.0029 & 0.0029 \\
\hline $52 \mathrm{PC}$ & 11 & 0.0032 & 0.0032 & 10.8 & 0.036 & 0.036 \\
\hline $56 \mathrm{PC}$ & 10.8 (D.B) & 0.165 & 0.165 & $10(\mathrm{D} . \mathrm{B})$ & 0.2 & 0.2 \\
\hline
\end{tabular}

•

D. B.-Drop in buffer due to the over flow. 


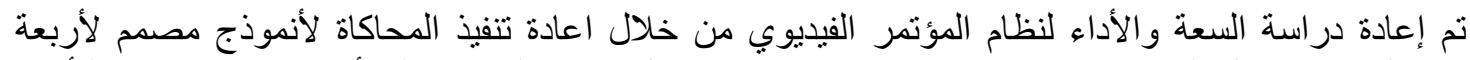

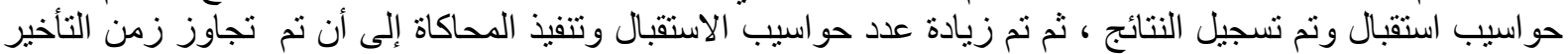

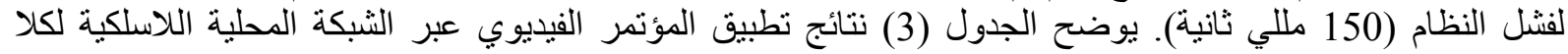
المعيارين IEEE 802.11a و IEEE 802.11g وبنمط الإرسال المتعدد.

5- - زيادة سعة الشبكة المحلية اللاسلكية : تم في هذا البحث اقتراح وسيلة جديدة لزيادة سعة نظام المؤتمر الفيديوي عبر الثبكة المحلية اللاسلكية ، وذللك بتبني معمارية جديدة لنقطة الوصول لتحسين أداء العطاء (improve throughput performance) و تقليل زمنة

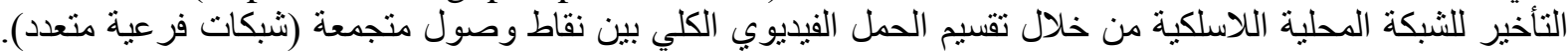

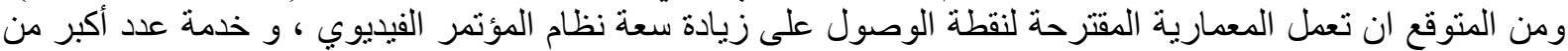

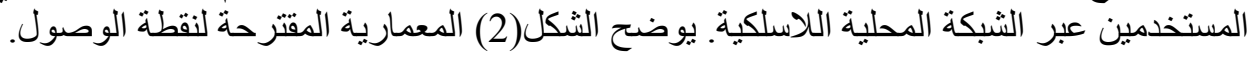

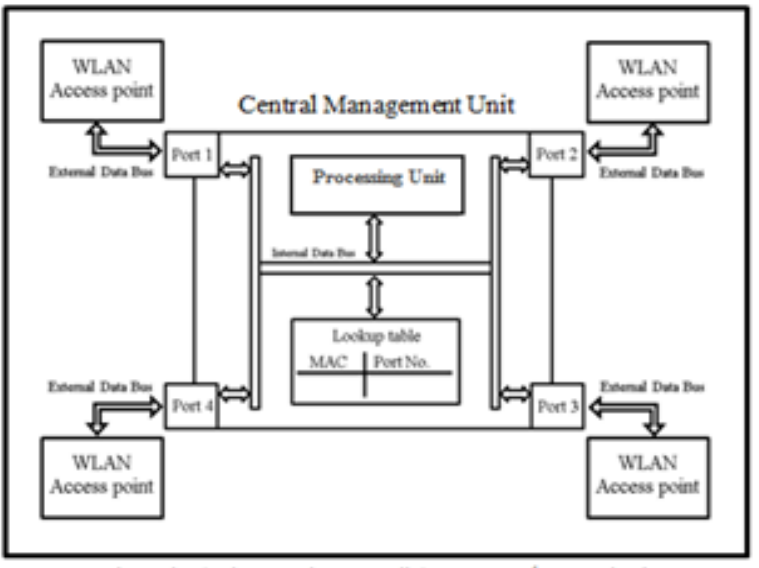

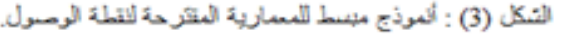

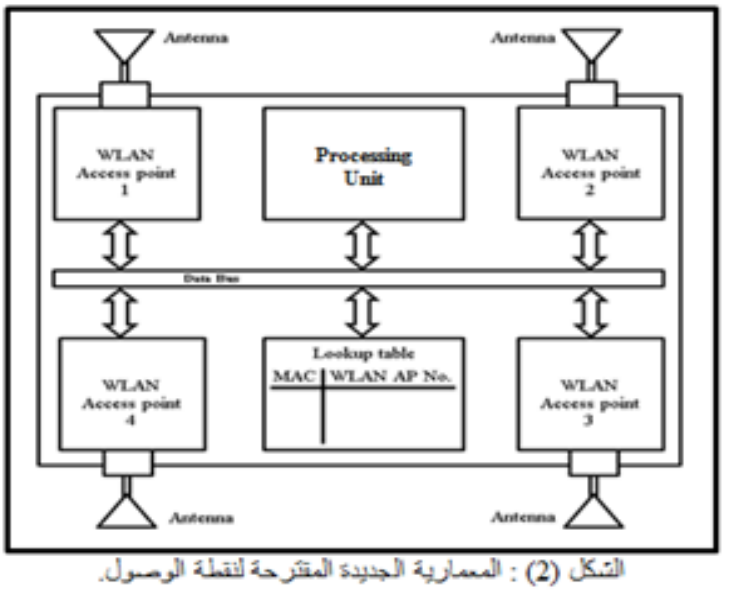

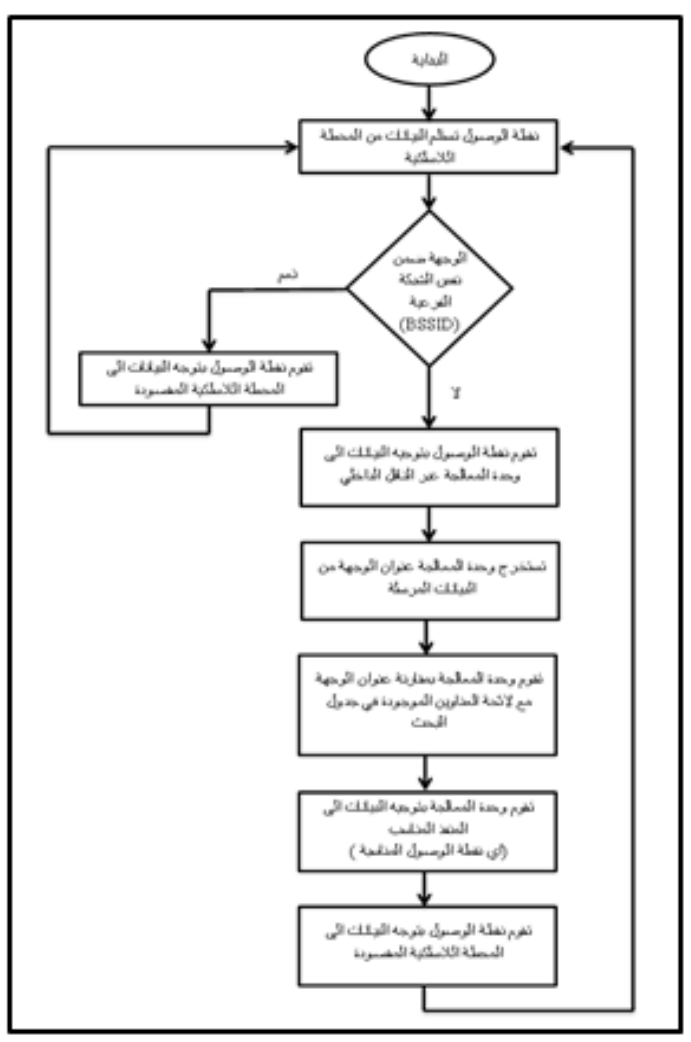

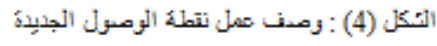

كل نقطة من نقاط الوصول اللاسلكية مسؤولة عن خدمة

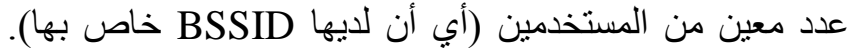
عند انتقال البيانات داخل نفس معرف مجمو عالفة الخدمة الاساسية

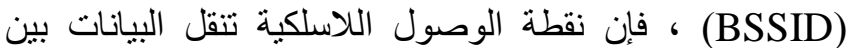

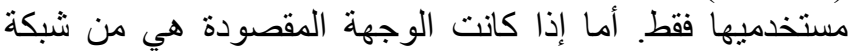

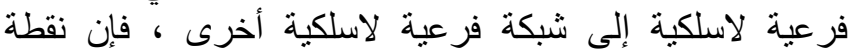

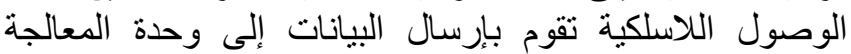

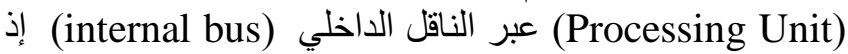

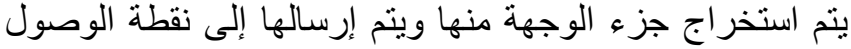
اللاسلكية المناسبة وفقا لجدول البحث (lookup table).

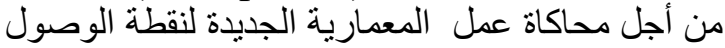

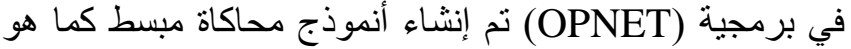

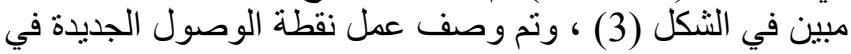

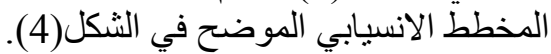

أـ الإرسال بالنمط الأحادي المتكرر باستخدام المعمارية الجديدة

\section{ل ل لنقطة الوصول:}

لحساب سعة نظام المؤتمر الفيديوي عبر الثبكة المحلية المبلية

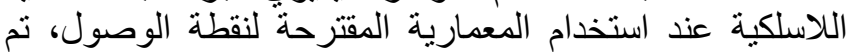

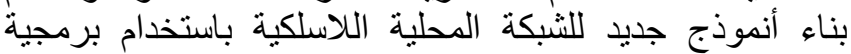
(OPNET Modeler 14.0) لنقطة الوصول. إذ نم استخدام أجهزة 


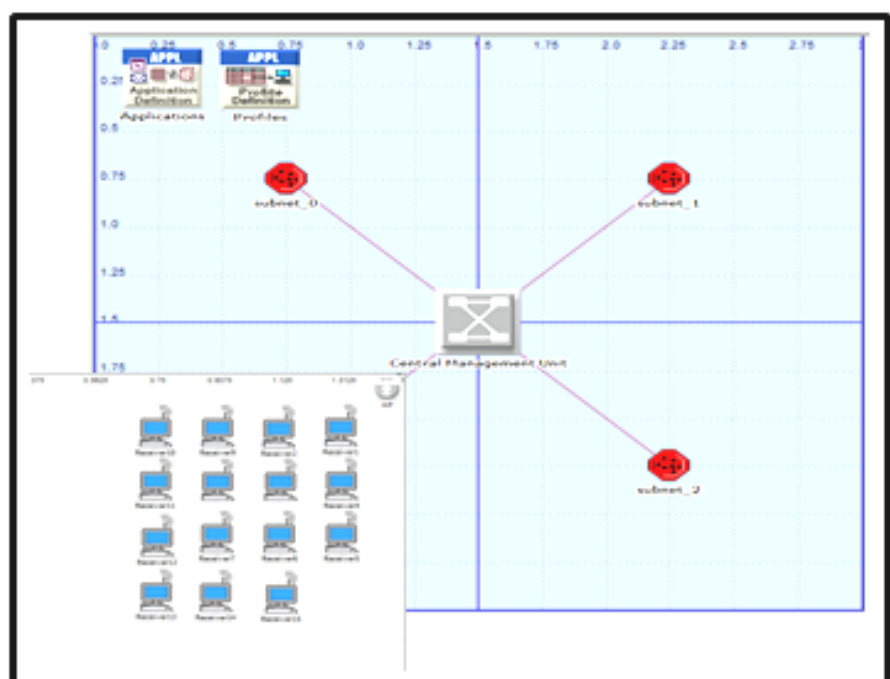

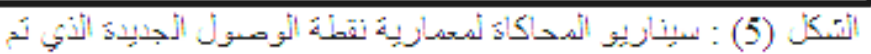

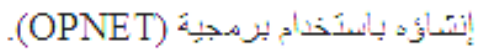

الثبكة والروابط المكافئة التي توجد في (14.0 بدلاً من مكونات معمارية نقطة الوصول التوليط ألمقترحة.

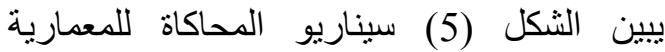
الجديدة لنقطة الوصول الني تم إنشاؤها باستخدام

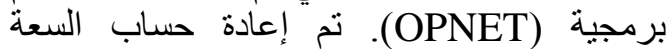
و الأداء لنظام المؤتمر الفيديوي عبر الثبكة المحلية

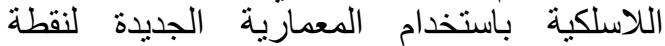

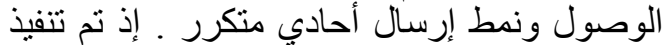
برنامج المحاكاة لأنموذج المادي المعمارية الجدريدة

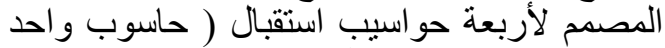

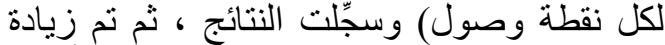
عدد حواسيب الاستقبال وتتفيذ المحاكاة إلى أن تم نمان

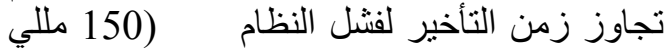
ثنانية).

يوضح الجدول (4) نتائج تطبيق المؤتمر

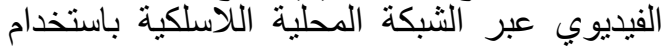
المعمارية الجديدة لنقطة الوصول و لكلا المعيارين

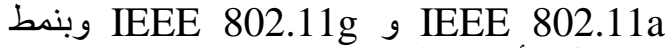
الإرسال الأحادي المتكرر.

الجدول (4) : نتائج تطبيق المؤتمر الفيديوي عبر الثبكة المحلية اللاسلكية باستخدام المعمارية الجديدة لنقطة الوصول لنمط الإرسال الأحادي المتكرر.

\begin{tabular}{|c|c|c|c|c|c|c|}
\hline \multirow{2}{*}{ PCNO. } & \multicolumn{3}{|c|}{ IEEE 802.11a } & \multicolumn{3}{|c|}{ IEEE $802.11 \mathrm{~g}$} \\
\hline & $\begin{array}{c}\text { MLAN Throughput } \\
\text { (Mibps) }\end{array}$ & MLAN Delay (Sec) & $\begin{array}{l}\text { Video Conference } \\
\text { Delay (Sec) }\end{array}$ & $\begin{array}{c}\text { WLAN Throughput } \\
\text { (Mbps) }\end{array}$ & MLAN Delay (Sec) & $\begin{array}{c}\text { Video Conference } \\
\text { Delay (Sec) }\end{array}$ \\
\hline $4 \mathrm{PC}$ & 1.49 & 0.00025 & 0.00047 & 1.52 & 0.00028 & 0.00064 \\
\hline $\mathbf{8} \mathbf{P C}$ & 2.95 & 0.00029 & 0.00056 & 3.1 & 0.00035 & 0.00071 \\
\hline $12 \mathrm{PC}$ & 4.4 & 0.00034 & 0.00063 & 4.2 & 0.00046 & 0.0008 \\
\hline $16 \mathrm{PC}$ & 5.6 & 0.0004 & 0.0007 & 5.6 & 0.0005 & 0.00088 \\
\hline $20 \mathrm{PC}$ & 7.2 & 0.00042 & 0.00072 & 7.2 & 0.00052 & 0.00092 \\
\hline $24 \mathrm{PC}$ & 8 & 0.00044 & 0.00074 & 8.3 & 0.00054 & 0.00095 \\
\hline $28 \mathrm{PC}$ & 10.2 & 0.00046 & 0.00075 & 10 & 0.00056 & 0.0098 \\
\hline $32 \mathrm{PC}$ & 12.4 & 0.00049 & 0.00077 & 12.4 & 0.00058 & 0.001 \\
\hline $36 \mathrm{PC}$ & 13.2 & 0.00052 & 0.00079 & 13.2 & 0.00062 & 0.0012 \\
\hline $40 \mathrm{PC}$ & 14 & 0.00056 & 0.00081 & 14 & 0.00066 & 0.00135 \\
\hline $44 \mathrm{PC}$ & 15.8 & 0.00057 & 0.00083 & 16.2 & 0.00071 & 0.0015 \\
\hline $48 \mathrm{PC}$ & 17.2 & 0.00059 & 0.00086 & 17 & 0.0011 & 0.0022 \\
\hline $52 \mathrm{PC}$ & 18.5 & 0.00062 & 0.00088 & 18.5 & 0.0012 & 0.0025 \\
\hline $56 \mathrm{PC}$ & 20.3 & 0.00065 & 0.0009 & 19.8 & 0.0019 & 0.0032 \\
\hline $60 \mathrm{PC}$ & 22.4 & 0.00069 & 0.00094 & 21.3 & 0.0033 & 0.0043 \\
\hline $64 \mathrm{PC}$ & 23.2 & 0.00073 & 0.0011 & 22.5 & 0.0042 & 0.005 \\
\hline $68 \mathrm{PC}$ & 24.5 & 0.00082 & 0.0012 & 23.9 (D.B) & 0.1 & 0.18 \\
\hline $72 \mathrm{PC}$ & 25.3 & 0.0009 & 0.0014 & & & \\
\hline $76 \mathrm{PC}$ & 28.2 & 0.00096 & 0.0016 & & & \\
\hline $80 \mathrm{PC}$ & 29.3 & 0.0014 & 0.0021 & & & \\
\hline 84 PC & 30 & 0.0015 & 0.0026 & & & \\
\hline $88 \mathrm{PC}$ & 32 & 0.0018 & 0.0035 & & & \\
\hline $92 \mathrm{PC}$ & 33 (D.B) & 0.05 & 0.06 & & & \\
\hline $96 \mathrm{PC}$ & 34 (D.B) & 0.06 & 0.1 & & & \\
\hline $100 \mathrm{PC}$ & 34 (D.B) & 0.1 & 0.22 & & & \\
\hline
\end{tabular}

ب- ألارسال بالنمط المتعدد باستخدام المعمارية الجديدة لنقطة الوصول:

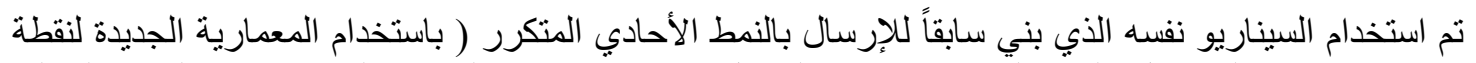
الوصول) بعد إعادة تهيئته للإرسال بالنمط المتعدد ـ يوضح الجدول (5) نتائج تطبيق المؤتمر الفيديوي عبر الثبكة المحلية 
اللاسلكية باستخدام المعمارية الجديدة لنقة الوصول و لكلا المعيارين IEEE 802.11a و IEEE 802.11g وبنمط

ت- ت تأثير عدد المشعات (نقاط الوصول) في أداء النظام:

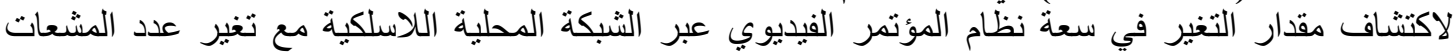

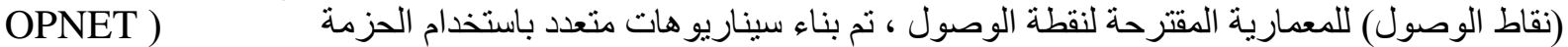

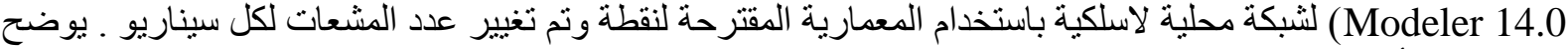

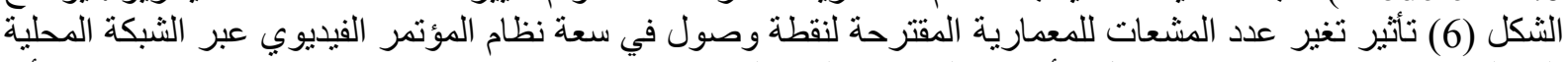

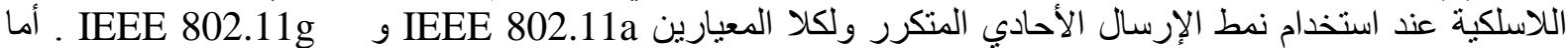

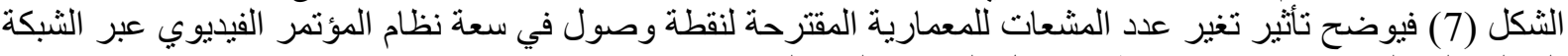
المحلية اللاسلكية عند استخدام نمط الإرسال المتعدد ولكلا المعيارين IEEE 802.11g.802.11a و IEEE 802.11a

الجدول (5) : نتائج تطبيق المؤتمر الفيديوي عبر الثبكة اللاسلكية المحلية باستخدام المعمارية الجديدة لنقطة الوصول لنمط الإرسال المتعدد.

\begin{tabular}{|c|c|c|c|c|c|c|}
\hline \multirow{2}{*}{ PC NO. } & \multicolumn{3}{|c|}{ IEEE 802.11a } & \multicolumn{3}{|c|}{ IEEE $\mathbf{8 0 2 . 1 1 \mathrm { g }}$} \\
\hline & $\begin{array}{l}\text { WLAN Throughput } \\
\text { (Mbps) }\end{array}$ & WLAN Delay (Sec) & $\begin{array}{c}\text { Video Conference } \\
\text { Delay (Sec) }\end{array}$ & $\begin{array}{c}\text { WLAN Throughput } \\
\text { (Mbps) }\end{array}$ & WLAN Delay (Sec) & $\begin{array}{c}\text { Video Conference } \\
\text { Delay (Sec) }\end{array}$ \\
\hline 4 PC & 1.25 & 0.00045 & 0.00085 & 1.25 & 0.00042 & 0.0008 \\
\hline $8 \mathrm{PC}$ & 2.4 & 0.001 & 0.0018 & 2.4 & 0.001 & 0.0016 \\
\hline $12 \mathrm{PC}$ & 3.5 & 0.0015 & 0.0024 & 3.5 & 0.0016 & 0.0025 \\
\hline $16 \mathrm{PC}$ & 4.6 & 0.002 & 0.0031 & 4.6 & 0.002 & 0.0033 \\
\hline $20 \mathrm{PC}$ & 5.8 & 0.0024 & 0.0037 & 5.75 & 0.0024 & 0.0036 \\
\hline 24 PC & 7 & 0.0025 & 0.0043 & 7 & 0.0025 & 0.0039 \\
\hline $28 \mathrm{PC}$ & 8.2 & 0.0032 & 0.0045 & 8.1 & 0.003 & 0.0046 \\
\hline $32 \mathrm{PC}$ & 9.3 & 0.0038 & 0.0056 & 9.3 & 0.0033 & 0.005 \\
\hline $36 \mathrm{PC}$ & 10.4 & 0.0042 & 0.006 & 10.4 & 0.0042 & 0.0062 \\
\hline $40 \mathrm{PC}$ & 11.5 & 0.0046 & 0.0069 & 11.5 & 0.0048 & 0.0075 \\
\hline 44 PC & 12.7 & 0.005 & 0.0075 & 12.7 & 0.005 & 0.008 \\
\hline $48 \mathrm{PC}$ & 13.8 & 0.0056 & 0.008 & 13.8 & 0.0055 & 0.0085 \\
\hline $52 \mathrm{PC}$ & 15 & 0.006 & 0.0086 & 15 & 0.006 & 0.0088 \\
\hline 56 PC & 16 & 0.0065 & 0.0092 & 16 & 0.007 & 0.0092 \\
\hline $60 \mathrm{PC}$ & 17 & 0.007 & 0.01 & 17 & 0.0082 & 0.01 \\
\hline 64 PC & 18.2 & 0.0076 & 0.0105 & 18.2 & 0.0085 & 0.0105 \\
\hline $68 \mathrm{PC}$ & 19.3 & 0.008 & 0.011 & 19.3 & 0.009 & 0.011 \\
\hline $72 \mathrm{PC}$ & 20.6 & 0.0085 & 0.0116 & 20.5 & 0.0093 & 0.0115 \\
\hline 76 PC & 21.9 & 0.009 & 0.012 & 21 & 0.0098 & 0.012 \\
\hline $80 \mathrm{PC}$ & 22.8 & 0.0095 & 0.0125 & 21.8 & 0.01 & 0.0125 \\
\hline 84 PC & 23.9 & 0.01 & 0.013 & 23 & 0.0105 & 0.0132 \\
\hline $88 \mathrm{PC}$ & 25 & 0.0105 & 0.0145 & 25 & 0.011 & 0.014 \\
\hline $92 \mathrm{PC}$ & 26 & 0.011 & 0.0155 & 25.8 & 0.012 & 0.016 \\
\hline 96 PC & 27 & 0.0115 & 0.016 & 26.9 & 0.014 & 0.018 \\
\hline $100 \mathrm{PC}$ & 28 & 0.012 & 0.0172 & 27.3 & 0.015 & 0.021 \\
\hline 104 PC & 29 & 0.0125 & 0.018 & 28.8 & 0.018 & 0.026 \\
\hline $108 \mathrm{PC}$ & 30 & 0.013 & 0.019 & 30 & 0.019 & 0.027 \\
\hline $112 \mathrm{PC}$ & 31 & 0.0135 & 0.02 & 31 (D.B) & 0.04 & 0.05 \\
\hline $116 \mathrm{PC}$ & 32 & 0.014 & 0.021 & 31.3 (D.B) & 0.14 & 0.16 \\
\hline $120 \mathrm{PC}$ & 34 & 0.0155 & 0.022 & & & \\
\hline $124 \mathrm{PC}$ & 35 & 0.016 & 0.0228 & & & \\
\hline $128 \mathrm{PC}$ & 36 & 0.0166 & 0.024 & & & \\
\hline $132 \mathrm{PC}$ & 37 & 0.017 & 0.026 & & & \\
\hline $136 \mathrm{PC}$ & 38 & 0.0173 & 0.027 & & & \\
\hline $140 \mathrm{PC}$ & 39 & 0.0175 & 0.0275 & & & \\
\hline 144 PC & 40 & 0.018 & 0.028 & & & \\
\hline 148 PC & 41.5 & 0.0183 & 0.0292 & & & \\
\hline 152 PC & 42.3 & 0.0195 & 0.03 & & & \\
\hline $156 \mathrm{PC}$ & 43 & 0.02 & 0.032 & & & \\
\hline $160 \mathrm{PC}$ & 43.6 (D.B) & 0.05 & 0.07 & & & \\
\hline 164 PC & 43.8 (D.B) & 0.08 & 0.09 & & & \\
\hline $168 \mathrm{PC}$ & 43.9 (D.B) & 0.13 & 0.153 & & & \\
\hline
\end{tabular}




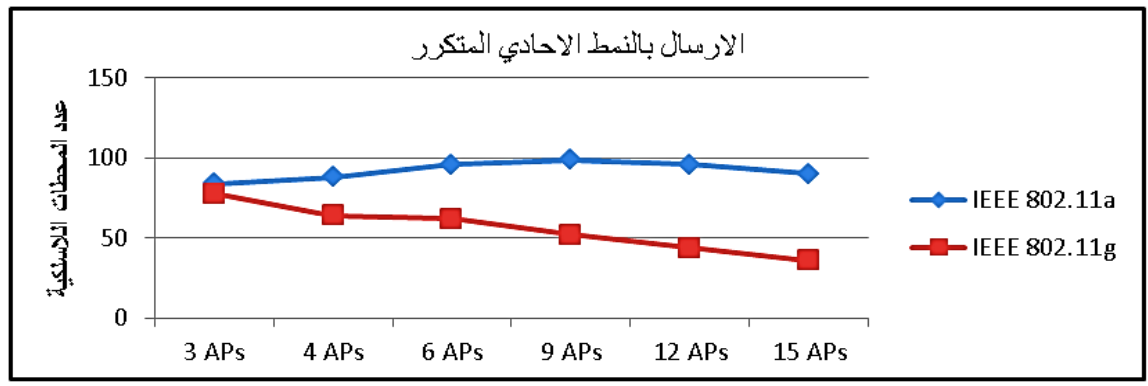

الثثكل (6) : ثأثير تغير عدد المشعات في سحة نظام المؤتمر الفيديوي لنمط الأرسال الاحادي المتكرر.

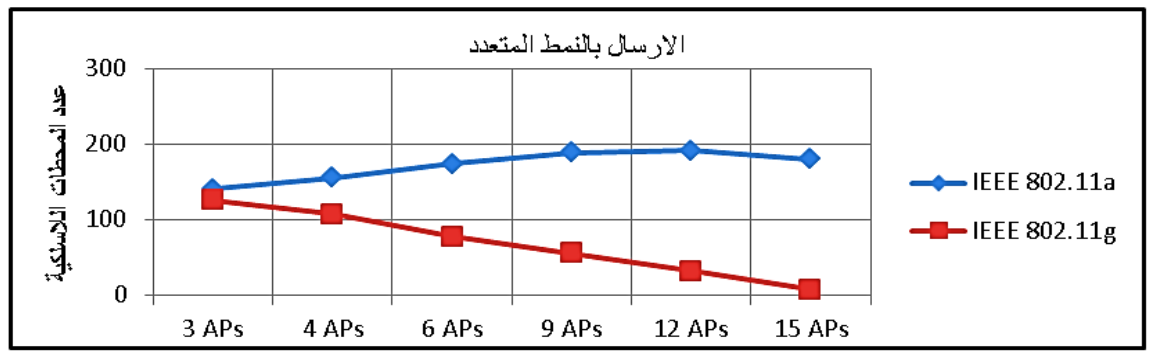

الثيكل (7) : تأثير تغير عدد المثنعات في سحة نظام المؤتمر الفيديوي لثمط الارسال المتحدد.

\section{تحقيق نظام المؤتمر الفيديوي عبر الشبكة اللاسلكية عملياً:}

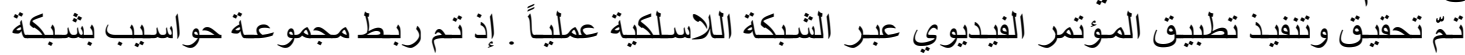

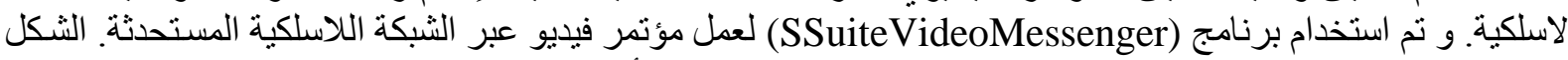

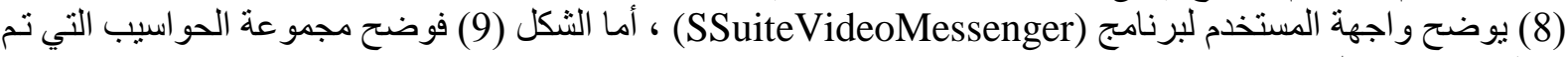
ربطها بشبكة لاسلكية.

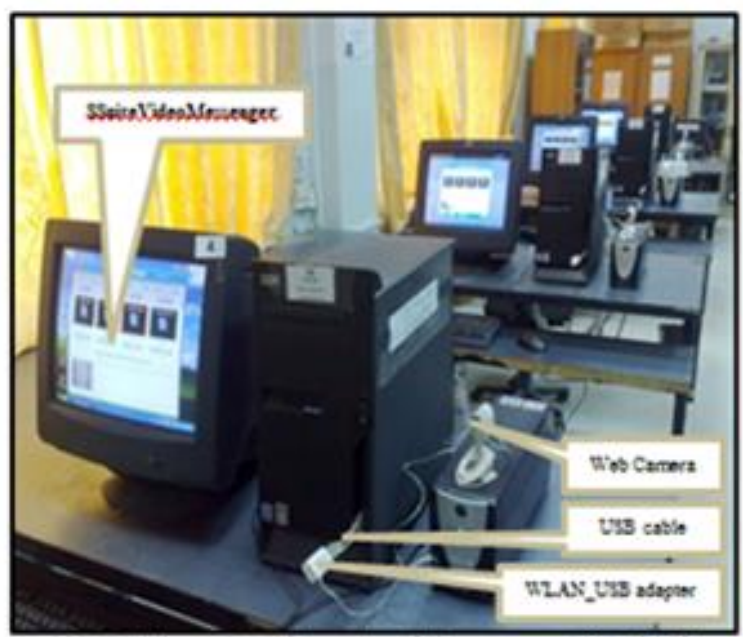

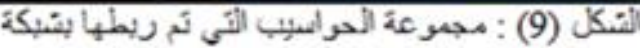

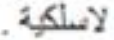

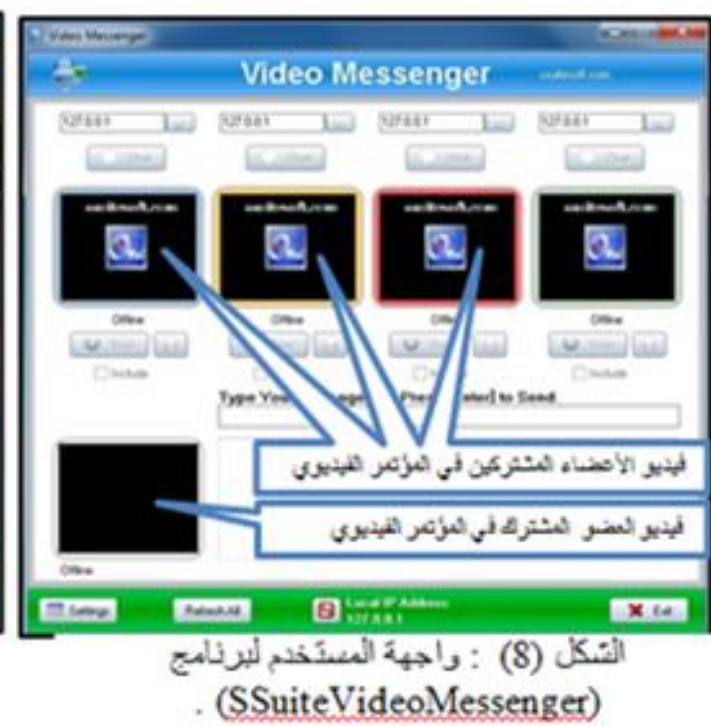

(SSuiteVideoMessenger)

تم حساب قيمة العطاء ومعدل زمن التأخير للمؤتمر الفيديوي عبر الثبكة اللاسلكية عند استخدام نقطسة الوصول

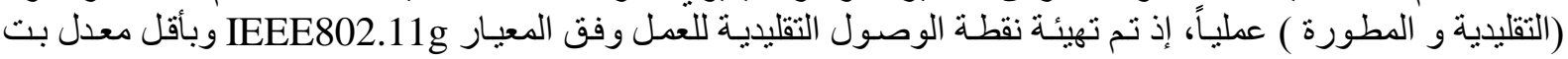

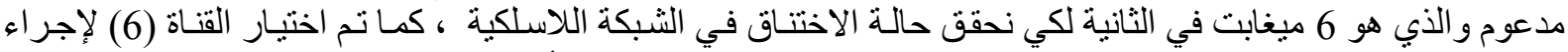

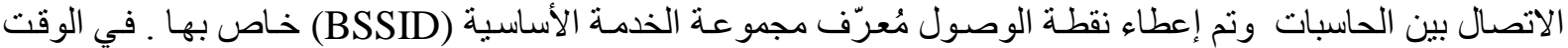
نفسه تم تهيئة ثلاثة نقاط وصول للعمل وفق المعيار IEEE802.11g وبأقل معدل بت مدعوم و الذي هو 6 ميغابت في الذي 


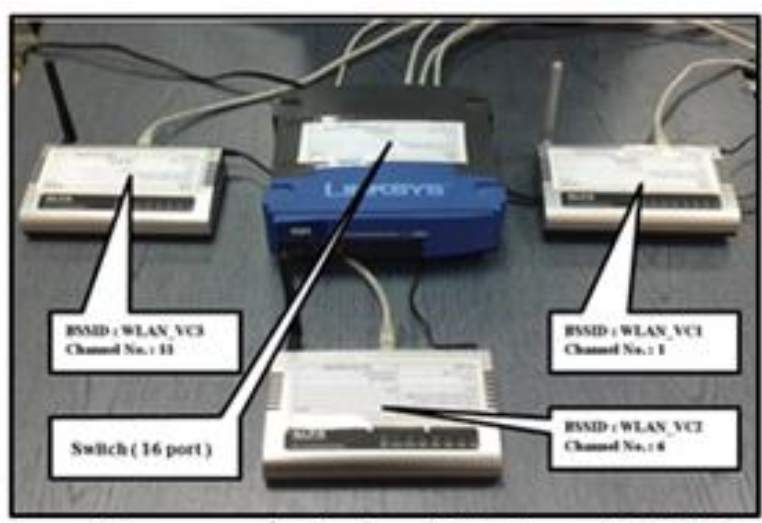

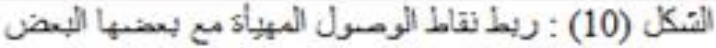
عير ميدل التيكة (Network Switch) بلفئخدام (UTP cable)

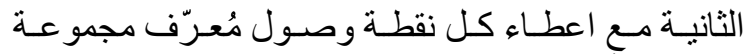

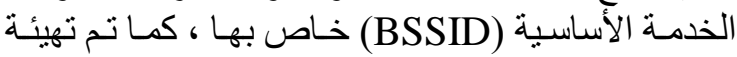

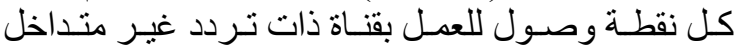

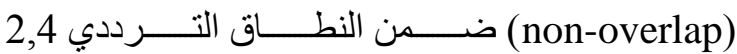

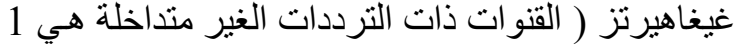

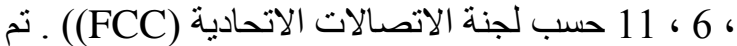
ربط نقاط الوصول المهيأة مع بعضها البعض الهض عبر مبدل الشبكة (Network Switch) باستخدام (UTP cable)

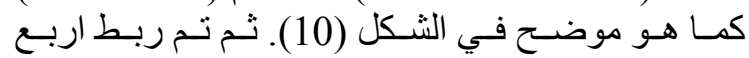

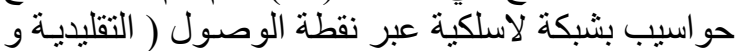

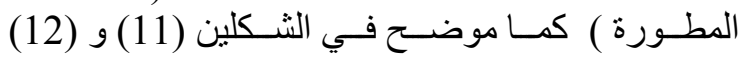

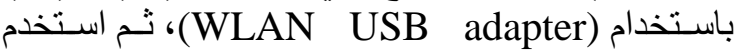

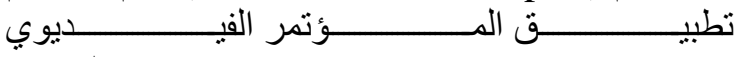

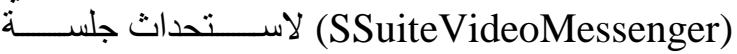
فيديوية مكونة من رئيس الجلسة و ثلاثة اعضاء عبر الثبكة اللاسلكية المصممة وبشكل مناظر للنماذج التي تم بنائها ببرنامج المحاكاة (OPNET).

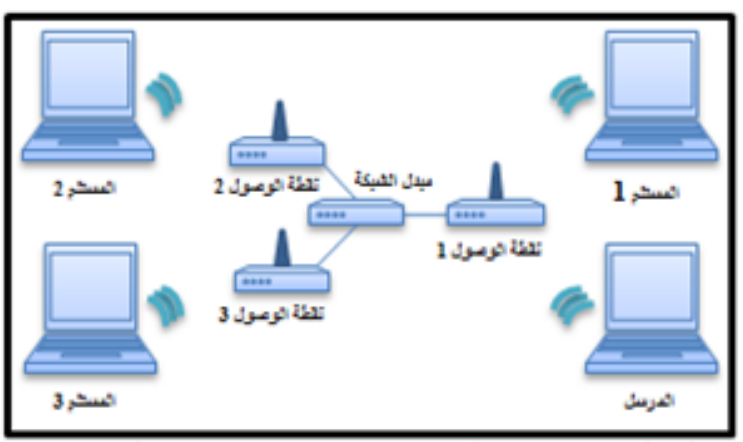

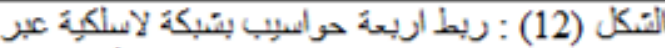

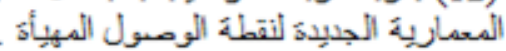

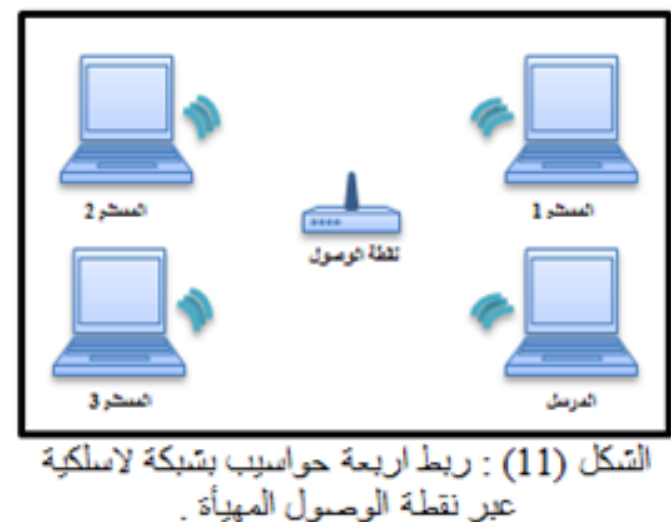

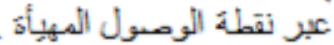

تم حساب قيمة العطاء ومعدل زمن التأخير للمؤتمر الفيديوي عبر الشبكة اللاسلكية عند استخدام نقطة الوصول (التقليدية و

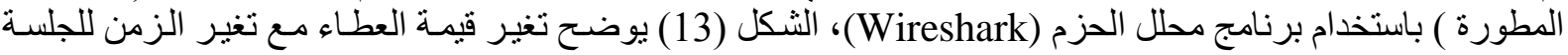

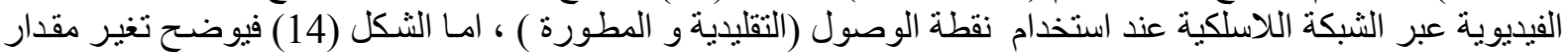

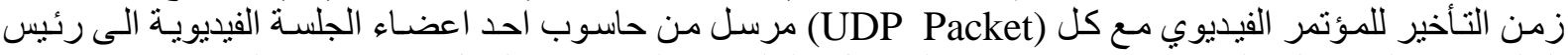

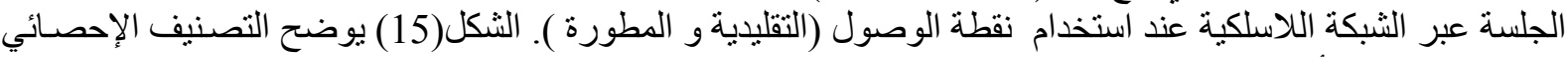

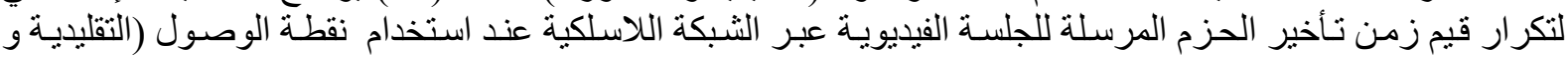

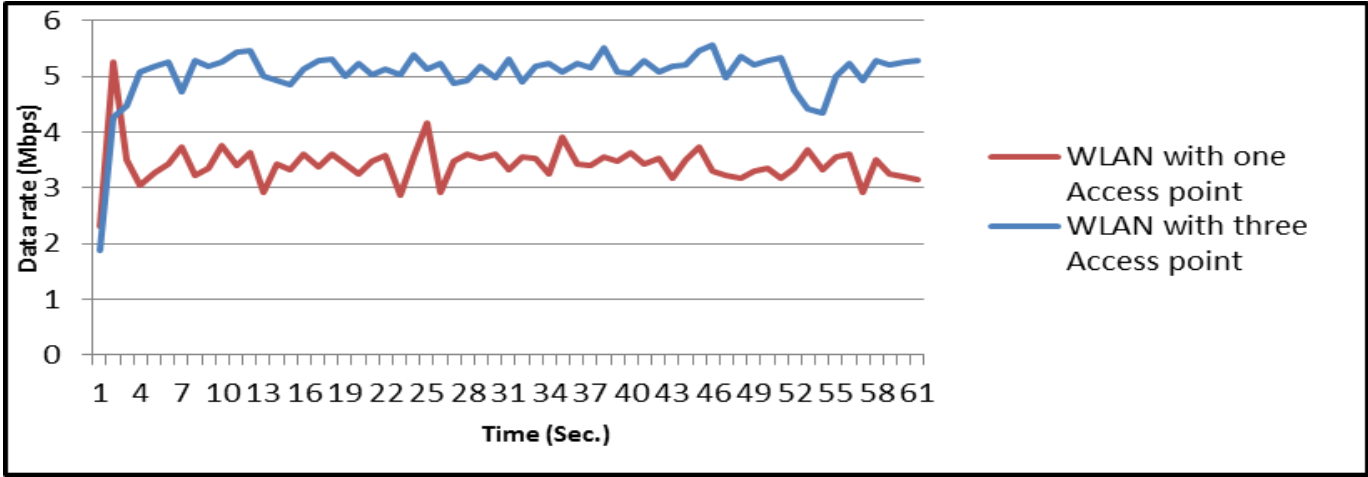

الثكل (13) : تغير قيمة العطاء مع تغير الزمن للجلسة الفيديوية عبر الثبكة اللاسلكية عند استخدام نقطة الوصول (التقليدية و المطورة المنية ). 
علي: دراسة بالمحاكاة لنظام المؤتمرات الفيديوية عبر الثبكة اللاسلكية مع التحقيق العملي

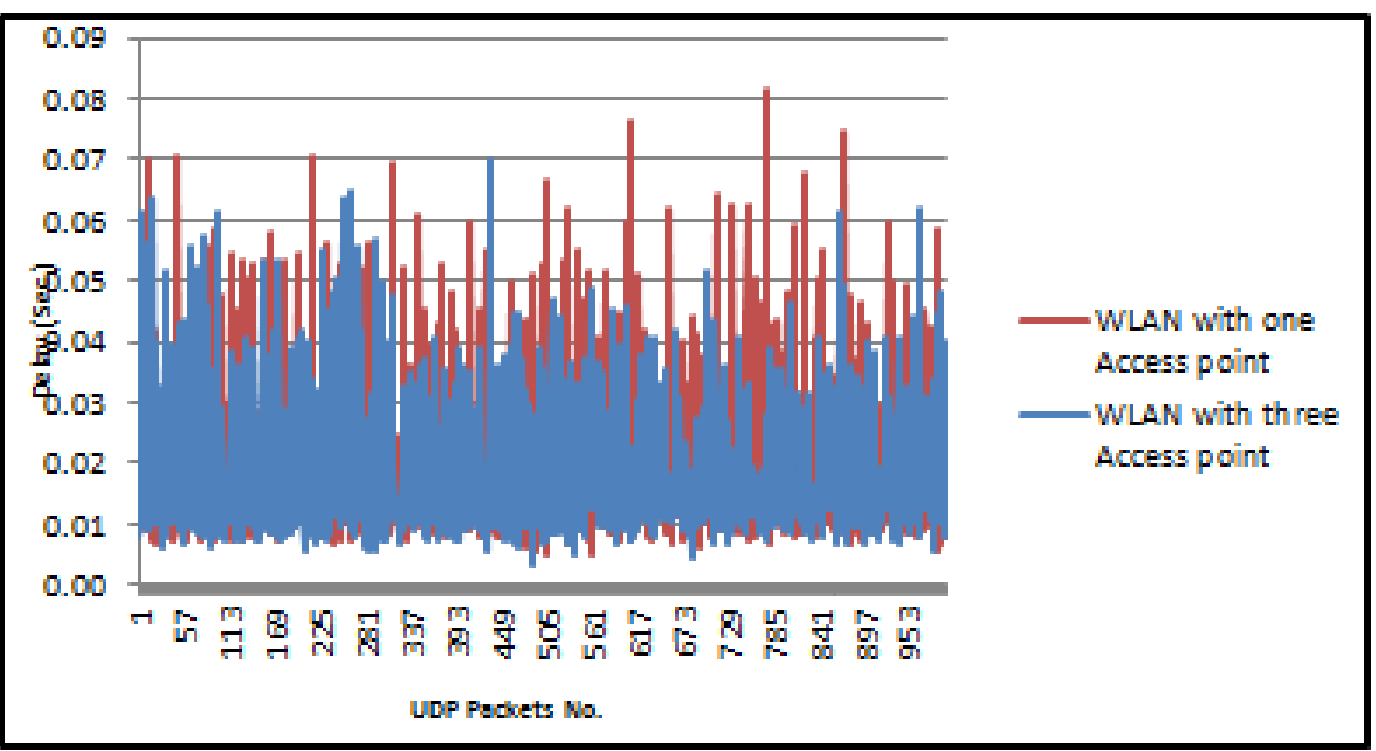

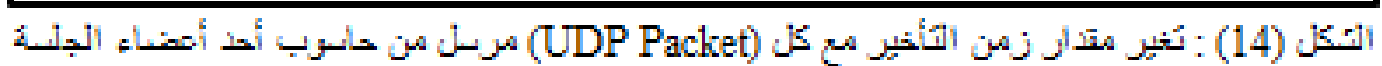

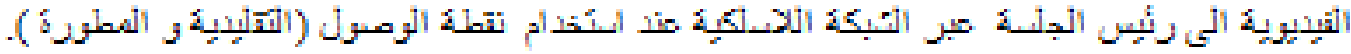

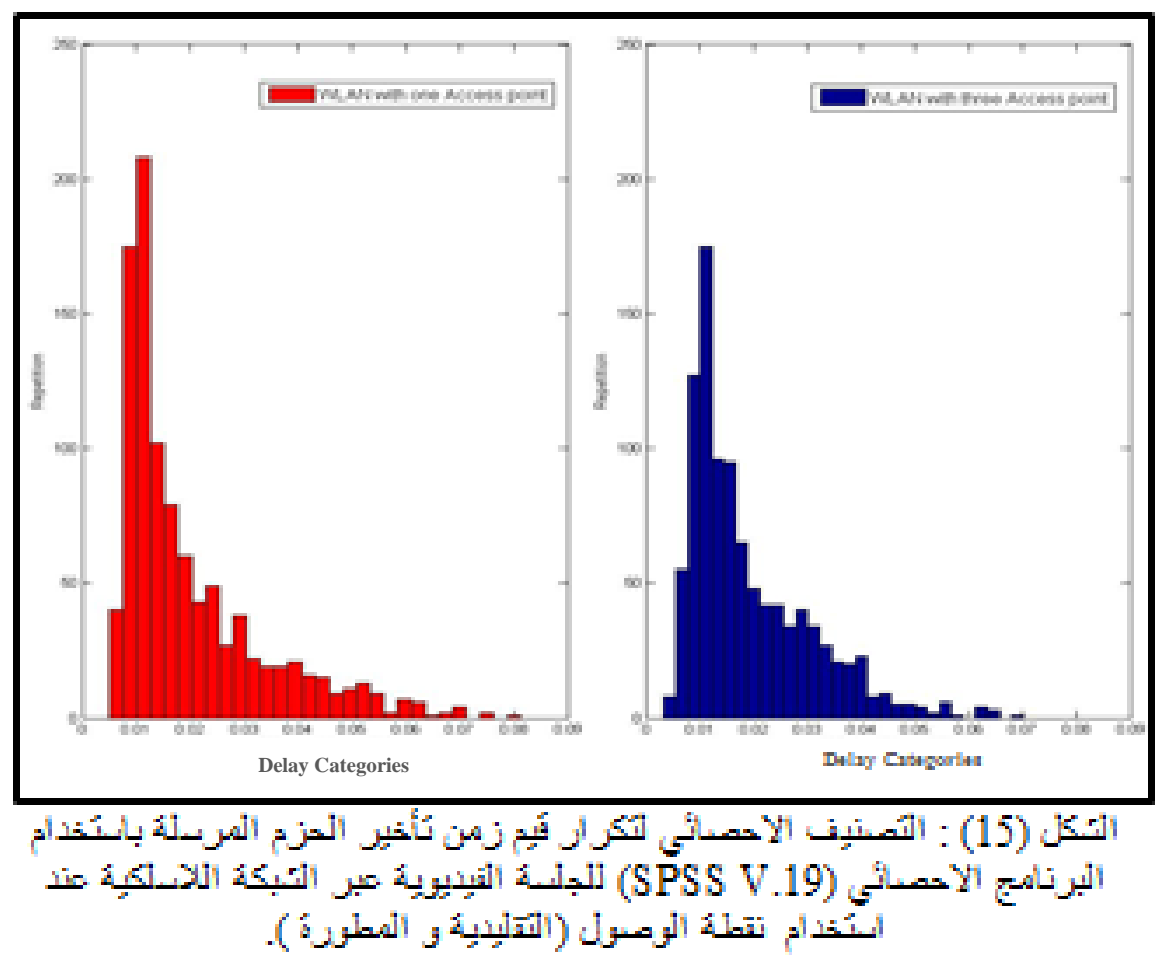

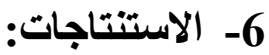

تم في هذا البحث اقتر اح معمارية جديدة لنقطة الوصول لزيادة سعة نظام المؤتمر الفيديوي عبر الثبكة المحلية

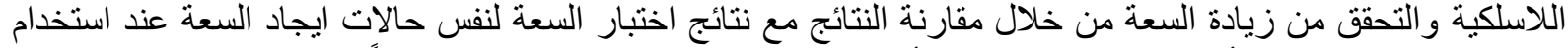
نقطة الوصول التقليدية. أظهرت نتائج المحاكاة أن المعيار IEEE 802.11a يدعم عدداً من المشتشركين في تطبيق

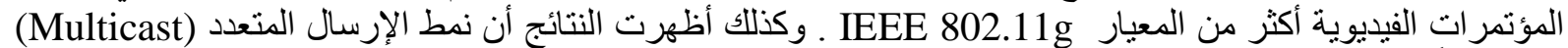
يدعم عدداً من المشتركين في تطبيق المؤتمر ات الفيديوية أكثر من نمط الإرسال الأحادي المتكرر (Multiple Unicast)

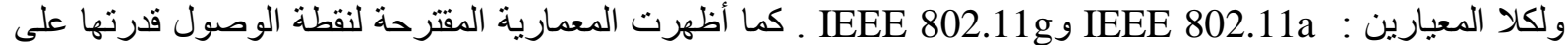
زيادة سعة نظام المؤتمرات الفيديوية عبر الثبكة المحلية اللاسلكية ، إذ اعتمدت الزيادة في السعة على نولية نوع المعيار 
اللاسلكي المستخدم و نمط الإرسال ـ إن هذا التحسن الواضح في السعة مَهد الطريق لاقتر اح جهاز جديد خاص بالثبكات اللاسلكية ألا وهو المبدل اللاسلكي المتعدد المنافذ والذي سيفتح الابواب لاستخدامات اضنافية للفية للشبكات المحلية اللاسلكية

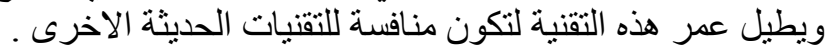

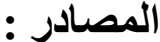

[1] R. Prasad and M. Ruggieri , "Technology trends in wireless communications" , Published by Artech House ,2003.

[2] H. Ki Kim , "Design and implementation of video conference system over the hybrid peer-topeer networks " , 12th WSEAS international conference on computers, Heraklion, Greece, July 23-25, 2008.

[3] E. Masala , C. F. Chiasserini , M. Meo and J. C. De Martin, "Real-time transmission of H.264 video over 802.11-based wireless ad hoc networks " Published by Springer, pp. 193-207, 2004.

[4] A. Holt and C. Huang, " 802.11 Wireless Networks Security and Analysis " , Published by Springer, 2011.

[5] M. Hossein ," Cross Layer interactions for adaptive communications in IEEE 802.11 wireless LANs", Doctoral Thesis , University of Nice - Sophia Antipolis , France, 2005.

[6] B. A. Forouzan, " Data Communications and Networking ", Published by McGraw-Hill , 2007.

[7] U. Sarwar, " Real time multiple codecs switching architecture for video conferencing ", M.Sc. Thesis, University of Sains, Malaysia, 2008.

[8] Video conferencing guide available at : www.mikogo.com/guide/video-conferencing .

[9] Z. Li , T. Herfet , " MAC Layer Support for High Rate Video Multicast Applications in Wireless LANs " , 7th International ITG Conference on Source and Channel Coding, pp. 1 - 6, 2008.

[10] Y. Ennaji, M. BouImaif and C. Alaoui , " Experimental Analysis of Video Performance over Wireless Local Area Networks ", IEEE International Conference on Multimedia Computing and Systems, pp. $488-494,2009$.

[11] A. Hidayat, C. Wilson , " Impact of Ad Hoc Network Parameters and Conditions on Video Conferencing Quality " , IEEE International Journal of Video \& Image Processing and Network Security, Vol. 11, pp. 21-27, 2011.

[12] A. H. Sadka, " Video Communications " , Published by John Wiley \& Sons, 2002.

[13] S. Bin Abdlatif , " Protocol design for real time multimedia communication over high-speed wireless networks ", Doctoral Thesis , Massey University, New Zealand , 2010. 